\title{
EL GRUPO FÓNICO Y EL GRUPO DE ENTONACIÓN EN EL ESPAÑOL HABLADO
}

\author{
ANTONIO Quilis. \\ Margarita Cantarero. \\ Manuel Esgueva.
}

Navarro Tomás hacia coincidir el grupo fónico con la unidad melódica cuando decía: "La unidad melódica es la porción mínima de discurso con sentido propio y con forma musical determinada. Los límites de la unidad melódica coinciden con los del grupo fónico" ${ }^{1}$. En el Manual de pronunciación, sólo habla de grupo fónico ${ }^{2}$, del que dice que es "la porción de discurso comprendida entre dos pausas o cesuras sucesivas de la articulación". Más tarde, define la unidad melódica en el Manual de entonación española ${ }^{3}$, como la "porción mínima de discurso con forma musical determinada, siendo al propio tiempo una parte por si misma significativa dentro del sentido total de la oración", y de nuevo señala que sus limites "coinciden en español con los del grupo fónico": pero una variante aparece aqui con relación a lo expuesto en otros trabajos: "las divisiones entre estos grupos o unidades no van siempre marcadas por verdaderas pausas. Con frecuencia el paso de una unidad a otra se manifiesta solamente por la depresión de la intensidad, por el retardamiento de la articulación y por el cambio más o menos brusco de la altura musical, sin que ocurra real y efectiva interrupción de las vibraciones vocálicas". Aparece, como se ve, una ligera contradicción entre los criterios delimitadores del grupo fónico y de la unidad melódica.

La extensión de la unidad melódica y, por lo tanto, del grupo fónico, según Navarro, calculada por medio del recuento realizado "en varias páginas

1 “El grupo fónico como unidad melódica”. RFH, I, 1939, 3-19. La cita, en la pág. 3.

2 21.a ed., Madrid, C. S. I. C., 1982, 29.

3 New York, Hispanic Institute on the United States, 1944, 13. 
de autores contemporáneos" ${ }^{4}$, arroja los siguientes resultados: a) la citada uniclad se extiende desde una hasta 18 silabas; b) "el cuerpo de la escala está claramente constituido por las unidades de cinco a 10 sílabas"; c) las unidades inferiores a cinco silabas sólo representan el $12,55 \%$ del total y las superiores a 10 silabas, el $19,54 \%{ }^{5}$; d) las unidades de mayor frecuencia son las de siete y ocho silabas; por si solas constituyen el $26,32 \%$.

1. Evidentemente, las pausas tienen una función delimitadora o demarcativa del enunciado; por necesidades sintácticas, fisiológicas, o ambas simultáneamente, es necesario realizar pausas cuando hablamos o leenos en voz alta. Mas esta función delimitadora se realiza muchas veces sólo por medio de la entonación, sin necesidad de pausa: una inflexión del fundamental $^{6}$ ascendente o descendente, delimita por si solo el enunciado; esta función delimitadora de la entonación ${ }^{7}$, acompañada o no de pausa, puede ser significativa ${ }^{8}$ o no; pero aun cuando no lo sea, es también necesaria para la descodificación e interpretación del mensaje; en español, aparece entre el sujeto y el predicado, entre sucesivos complementos, o después de complementos hiperbatizados, entre los distintos grupos que configuran la enumeración, etc.

El enunciado Platero es pequeño, peludo, suave puede tener pausas entre los distintos miembros de la enumeración.

\section{[1] platero es pequeño $\downarrow$ \# peludo $\downarrow$ \# suave $\downarrow$ \#}

pero, si fuese impausado, las palabras que integran esta enumeración estarían delimitadas sólo por la inflexión del fundamental:

\section{[2] Platero es pequeño $\downarrow$ peludo $\downarrow$ suave $\downarrow$ \#.}

El enunciado [1] consta de tres grupos fónicos, delimitados por la entonación y la pausa. El [2] tiene tres grupos, delimitados sólo por la entonación, y todo él constituye un grupo fónico.

Cada uno de los tres grupos del enunciado [2] es un grupo de entonación, definido por Quilis ${ }^{9}$ como "la porción de discurso comprendida entre dos

\footnotetext{
- Unamuno, Baroja, Azorín, Ayala, Miró.

5 "El grupo fónico", pág. 7.

- También llamado, aunque no muy apropiadamente, tono.

7 Véase Antonio Quilis: Fonética acústica de la Lengua española, Madrid, Gredos, $10^{\mathrm{a}}$ reimpresión, $1^{\mathrm{a}}{ }^{\mathrm{a}}$ edición, 1988, págs. 387-389 y 422-437.

8 En casos, por ejemplo, como: Juan pregunta quién va a entrar / Juan pregunta: ¿quién va a entrarl / Juan: pregunta quién va a entrar; o $2 \times(3+1) /(2 \times 3)+1$.

- A. Quilis: Tratado de Fonología y Fonética españolas, Madrid, Gredos, 1993, $\S 14.4 .1$ y 14.4 .2 .
} 
pausas, entre pausa e inflexión del fundamental, entre inflexión del fundamental y pausa o entre dos inflexiones del fundamental, que configuran una unidad sintáctica más o menos larga o compleja (sintagma, cláusula, oración)".

En [1], los grupos de entonación coinciden con los grupos fónicos: hay inflexión del fundamental y pausa; en [2], hay tres grupos de entonación en un solo grupo fónico.

Junto al grupo de entonación, seguimos manteniendo el concepto de grupo fónico ${ }^{10}$. Pensamos que ambos son necesarios para la descripción de los rasgos suprasegmentales de la lengua hablada.

2. Los datos que utilizamos en este trabajo proceden de los materiales de la lengua española hablada en Madrid. Hemos analizado, en el SonaGraph 7800 de la Kay Elemetrics Co., 3.000 espectrogramas, correspondientes a 12 informantes, de distintas generaciones y sexos, en estilos de conversación diferentes: un grupo, de ocho, corresponde" al estilo que denominamos diálogos dirigidos; el otro grupo, de cuatro informantes ${ }^{12}$, corresponde a los diálogos espontáneos ${ }^{13}$. En estos materiales, hemos estudiado: a) el comportamiento del fundamental, es decir, dónde presentaba alguna inflexión con función delimitadora; b) las duraciones de las pausas, de los grupos fónicos, de los grupos de entonación y de las sílabas; c) el número de sílabas en los grupos de entonación. Los resultados de nuestros análisis son los siguientes:

\section{El grupo fónico.}

Vamos a exponer a continuación los datos correspondientes a los grupos fónicos.

10 Y su definición tradicional: "porción de discurso comprendida entre dos pausas".

11 Son los que en los cuadros aparecen del siguiente modo: $1^{\mathrm{a}} \mathrm{V}$ : primera generación, varón; $1{ }^{\mathrm{a}} \mathrm{M}$ : primera generación, mujer; . $^{\mathrm{a}} \mathrm{V}$ : segunda generación, varón; 2. ${ }^{\mathrm{a}} \mathrm{M}$ : segunda generación, mujer; etc.

${ }_{13} 1^{\mathrm{a}} \mathrm{Ve}$ : primera generación, varón; $10^{\mathrm{a}} \mathrm{Me}$ : primera generación, mujer; $4 .^{\mathrm{a}} \mathrm{Vd}$ : cuarta generación, varón; $2^{\mathrm{a}} \mathrm{Md}$ : segunda generación, mujer.

13 Sobre esta investigación dimos cuenta en el trabajo: Antonio Quilis, Margarita Cantarero, Manuel Esgueva, "La investigación sobre la fonética de la norma lingüística culta de la Lengua española hablada en Madrid". Actes du XVII '̇me Congrès International de Linguistique et Philologie Romanes, vol. 3, 1985, págs. 67-77. (Aix-enProvence, 29 août - 3 septembre 1983.) 


\subsection{Número de silabas del grupo fónico.}

El número de grupos fónicos es lógicamente muy elevado y muy heterogéneo, aunque, en general, con una graduación clara, en todos los informantes. En el cuadro 1, of́recemos los valores de los diálogos dirigidos: las medias de grupos fónicos ( $\overline{\mathrm{X}}$ en el cuadro), para los ocho informantes, según el número de sílabas:

Cuadro 1.

\begin{tabular}{|c|c|c|c|}
\hline $\mathrm{N}^{\circ}$ de sílabas & $\bar{x}$ & N. ${ }^{\circ}$ de sílabas & X \\
\hline 1 & 47 & 27 & 2.7 \\
\hline 2 & 37 & 28 & 3 \\
\hline 3 & 36,8 & 29 & 2,5 \\
\hline 4 & 40,75 & 30 & 2,6 \\
\hline 5 & 37 & 31 & 2.3 \\
\hline 6 & 33 & 32 & 2,6 \\
\hline 7 & 28 & 33 & 2,2 \\
\hline 8 & 30,6 & 34 & 2,6 \\
\hline 9 & 25 & 35 & 1,5 \\
\hline 10 & 24,5 & 36 & 2 \\
\hline 11 & 18,8 & 37 & 2 \\
\hline 12 & 13,8 & 38 & 1 \\
\hline 13 & 16 & 39 & 1,5 \\
\hline 14 & 16 & 40 & 1,5 \\
\hline 15 & 13 & 41 & 1,2 \\
\hline 16 & 10 & 42 & 1,2 \\
\hline 17 & 7,5 & 43 & 2 \\
\hline 18 & 8,3 & 44 & 1 \\
\hline 19 & 8,2 & 45 & 2 \\
\hline 20 & 6,8 & 46 & 1 \\
\hline 21 & 6,5 & 47 & 1 \\
\hline 22 & 6 & 48 & 1 \\
\hline 23 & 3,7 & 49 & 2 \\
\hline 24 & 2 & 50 & 1 \\
\hline 25 & 3,5 & 53 & 1 \\
\hline 26 & 4 & & \\
\hline
\end{tabular}

De los datos de este cuadro, se deduce que: a) los valores medios más altos de los grupos fónicos se extienden desde los que tienen una sílaba, el más numeroso, hasta los de 16 sílabas: b) El orden descendente es el siguiente : 


\section{Cuadro 2.}

\begin{tabular}{|c|c|c|c|c|c|c|}
\hline $1 .^{\circ}$ & Grupos & fónicos & de & & sílaba & $=47$ \\
\hline $2^{\circ}$ & " & $"$ & $"$ & 4 & sílabas & $=40,7$ \\
\hline \multirow[t]{2}{*}{$30^{\circ}$} & " & 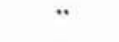 & $\cdot$ & 2 & " & $=37$ \\
\hline & $"$ & $"$ & $"$ & 5 & $"$ & $=37$ \\
\hline $4^{\circ}$ & $"$ & $"$ & $"$ & 3 & $\cdot$ & $=36,8$ \\
\hline $5 .^{\circ}$ & " & 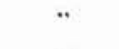 & $"$ & 6 & "* & $=33$ \\
\hline $6^{\circ}$ & $"$ & 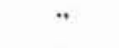 & . & 8 & $\cdot$. & $=30,6$ \\
\hline $7 .^{\circ}$ & $"$ & .. & . & 7 & $\cdot$ & $=28$ \\
\hline $8^{\circ}$ & $\cdot$ & $\cdot$ & $"$ & 9 & *. & $=25$ \\
\hline $9 .^{\circ}$ & $"$ & $\cdot$ & ". & 10 & *. & $=24.5$ \\
\hline $10 .^{\circ}$ & $"$ & ". & $"$ & 11 & " & $=18.8$ \\
\hline \multirow[t]{2}{*}{$11^{\circ}$} & $"$ & 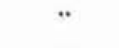 & 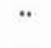 & 13 & .. & $=16$ \\
\hline & $\cdot$ & $\cdot$ & $"$ & 14 & * & $=16$ \\
\hline $12^{\circ}$ & $"$ & $*$ & $*$ & 12 & 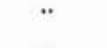 & $=13,8$ \\
\hline $13 .^{\circ}$ & $"$ & " & " & 15 & $*$ & $=13$ \\
\hline $14^{\circ}$ & $"$ & $"$ & $\cdot$ & 16 & " & $=10,2$. \\
\hline
\end{tabular}

c) El 50,89 de los 16 grupos dados anteriormente corresponden a grupos fónicos de una a cinco sílabas. d) Los grupos fónicos más numerosos son los de una y cuatro sílabas. e) Estas cifras son radicalmente distintas de las dadas para la lengua literaria.

En el cuadro 3, representamos los mismos valores, correspondientes a los diálogos espontáneos. En este caso, como se recordará, el número de informantes es cuatro.

Cuadro 3.

\begin{tabular}{|c|c|c|c|}
\hline $\mathrm{N}^{\circ}{ }^{\circ}$ de sílabas & $\bar{x}$ & N. ${ }^{\circ}$ de sílabas & $\overline{\mathrm{x}}$ \\
\hline 1 & 13,7 & 13 & 6 \\
\hline 2 & 14,2 & 14 & 8,2 \\
\hline 3 & 16,2 & 15 & 5,5 \\
\hline 4 & 18 & 16 & 7,5 \\
\hline 5 & 18,7 & 17 & 3,7 \\
\hline 6 & 18,5 & 18 & 7,2 \\
\hline 7 & 14,5 & 19 & 4 \\
\hline 8 & 15 & 20 & 2,2 \\
\hline 9 & 10,7 & 21 & 1,7 \\
\hline 10 & 11,5 & 22 & 2,7 \\
\hline 11 & 8,7 & 23 & 2,2 \\
\hline 12 & 8.7 & 24 & 3 \\
\hline
\end{tabular}


Cundro 3 (continuación).

\begin{tabular}{cllll}
\hline$N^{\circ}$ de silabas & $\mathrm{X}$ & & $\mathrm{N} .^{\circ}$ de silabas & $\mathrm{X}$ \\
\cline { 4 - 4 } 25 & 2 & & 33 & 1 \\
26 & 2 & & 34 & 1 \\
27 & 1 & 35 & 2 \\
28 & 1 & & 37 & 1 \\
29 & 1 & 38 & 1 \\
30 & 2 & 41 & 1,5 \\
31 & 1,5 & 44 & 1 \\
32 & 1 & 48 & 1 \\
\hline
\end{tabular}

Los resultados son diferentes de los del cuadro anterior. En orden descendente, los valores de los 16 grupos de medias más altas son los siguientes:

\section{Cuadro 4.}

\begin{tabular}{|c|c|c|c|c|c|c|}
\hline $1 .^{\circ}$ & Grupos & fónicos & de & 5 & ílabas & $=18,7$ \\
\hline $20^{\circ}$ & $"$ & $"$ & $"$ & 6 & $"$ & $=18,5$ \\
\hline $30^{\circ}$ & .. & . & $*$ & 4 & 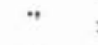 & $=18$ \\
\hline $4 .^{\circ}$ & $"$ & $"$ & 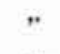 & 3 & $"$ & $=16,2$ \\
\hline $50^{\circ}$ & $"$ & 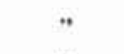 & $"$ & 8 & $*$ & $=15$ \\
\hline $6^{\circ}$ & $"$ & $"$ & $"$ & 7 & $"$ & $=14,5$ \\
\hline $70^{\circ}$ & $"$ & $"$ & $"$ & 2 & $"$ & $=14,2$ \\
\hline $8^{\circ}$ & $*$ & $"$ & $"$ & 1 & .* & $=13.7$ \\
\hline $9 .^{\circ}$ &. &. & $n$ & 10 & $"$ & $=11,5$ \\
\hline $100^{\circ}$ & $"$ & $"$ & $"$ & 9 & $"$ & $=10,7$ \\
\hline \multirow[t]{2}{*}{$11^{\circ}$} & $"$ & $"$ & $"$ & 11 & $"$ & $=8,7$ \\
\hline & $"$ & $*$ & $"$ & 12 & $"$ & $=8,7$ \\
\hline $12 .^{\circ}$ & $*$ & " & $"$ & 14 & $"$ & $=8,2$ \\
\hline $13 .^{\circ}$ & $"$ & $"$ & $"$ & 16 & $"$ & $=7,5$ \\
\hline $14 .^{\circ}$ & $"$ & $"$ & $"$ & 18 & $"$ & $=7,2$ \\
\hline $15^{\circ}$ & $"$ & r & $"$ & 13 & $"$ & $=6$ \\
\hline
\end{tabular}

a) $\mathrm{El} 52,2 \%$ de estos 16 grupos corresponden a los grupos fónicos de tres, cuatro, cinco, seis, siete y ocho sílabas, siendo los más numerosos los de cinco, seis y cuatro, en este orden. Estos datos también son muy diferentes de los de la lengua literaria; b) los distintos estilos entre ambos tipos de encuesta originan las diferencias señaladas. 
3.2. Las columnas de los cuadros 5 y 6 ofrecen los valores que a continuación indicamos, referidos a las encuestas dirigidas, en el $5, \mathrm{y}$ a las espontáneas, en el 6 .

1. Media del número de sílabas por grupo fónico.

2. Duración media del grupo fónico, en centésimas de segundo.

3. Duración media de la pausa, en centésimas de segundo.

4. Duración media de la sílaba, en centésimas de segundo.

Cladro 5.

\begin{tabular}{|c|c|c|c|c|c|c|c|}
\hline & Gen. & & & 1 & 2 & 3 & 4 \\
\hline $1 .^{\mathrm{a}} \mathrm{V}$ & $\ldots$ & $\ldots$ & $\ldots$ & 7.9 & 112,6 & 37 & 17,8 \\
\hline 1. ${ }^{\mathrm{a}} \mathrm{M}$ & $\ldots$ & $\ldots$ & $\ldots$ & 13,7 & 150 & 35 & 12,3 \\
\hline $2 .^{\mathrm{a}} \mathrm{V}$ & $\ldots$ & $\ldots$ & $\ldots$ & 8,2 & 122,6 & 42 & 19,7 \\
\hline 2. ${ }^{\mathrm{a}} \mathrm{M}$ & $\ldots$ & $\ldots$ & $\ldots$ & 9,7 & 124 & 43,5 & 15,3 \\
\hline 3. ${ }^{\mathrm{a}} \mathrm{V}$ & $\ldots$ & $\ldots$ & $\ldots$ & 6,4 & 95,3 & 49,3 & 19 \\
\hline $3 .^{\mathrm{a}} \mathrm{M}$ & $\ldots$ & $\ldots$ & $\ldots$ & 12,8 & 165 & 50 & 14 \\
\hline $4 .^{\mathrm{a}} \mathrm{V}$ & $\ldots$ & $\ldots$ & $\ldots$ & 7 & 103 & 49 & 14,7 \\
\hline $4 .^{\mathrm{a}} \mathrm{M}$ & $\ldots$ & $\ldots$ & $\ldots$ & 10.7 & 127,4 & 70 & 12,4 \\
\hline$\overline{\mathrm{x}} \ldots$ & $\ldots$ & $\ldots$ & $\ldots$ & 9,5 & 124,98 & 46,9 & 15,6 \\
\hline
\end{tabular}

Cuadro 6.

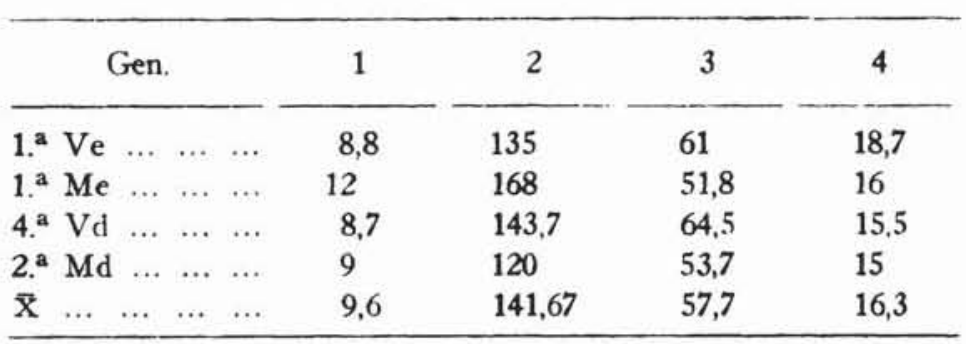

De ellos, podemos deducir que: a) la media total del número de sílabas por grupo fónico es de 9,5 ; b) las mujeres superan en la media del número de sílabas por grupo fónico a los hombres; c) la duración media de los grupos fónicos es de 133,3 c. s.; d) en general, las mujeres superan en la media de la duración del grupo fónico a los hombres; e) la duración media de la pausa es de 50,5 c. s.; f) la duración media de la silaba es de 15,8 c. s. : g) la duración media de las silabas es mayor en los hombres que en las mujeres.

\section{El grupo de entonación.}

Los datos correspondientes al grupo de entonación son los siguientes: 
4.1. Número de silabas del grupo de entonación.

1.a variedad de grupos de entonación, en cuanto al número de silabas que integran su composición no es tan grande como la de grupos fónicos; en el cuadro 7, aparecen los valores de los diálogos dirigidos: representamos los valores de las medias de los grupos de entonación de los ocho informantes, según el número de sílabas; al mismo tiempo, los ordenamos de mayor a menor.

Clianko 7.

\begin{tabular}{|c|c|c|c|}
\hline $\mathrm{N}^{\circ}$ de silabas & $\overline{\mathrm{x}}$ & $\mathrm{N} .^{\circ}$ de sílabas & $\overline{\mathrm{x}}$ \\
\hline 1 & 129,8 & 15 & 5,7 \\
\hline 4 & 127,5 & 14 & 5,5 \\
\hline 3 & 114,8 & 16 & 5,3 \\
\hline 2 & 109,7 & 18 & 3 \\
\hline 5 y 6 & 85,8 & 17 & 1,8 \\
\hline 7 & 62,2 & 21 & 1,5 \\
\hline 8 & 56.2 & 19 & 1,4 \\
\hline 9 & 40,7 & 20 & 1 \\
\hline 10 & 32,7 & 22 & 1 \\
\hline 11 & 20,5 & 23 & 1 \\
\hline 12 & 14,7 & 26 & 1 \\
\hline 13 & 10,1 & 34 & 1 \\
\hline
\end{tabular}

Como puede observarse, el $57,7 \%$ de los grupos de entonación están compuestos, en este orden, por una, cuatro, tres y dos silabas. En general, se mantienen las mismas tendencias que en los grupos fónicos.

En el cuadro 8, damos los nismos valores, para los diálogos espontáneos:

Cuadro 8.

\begin{tabular}{|c|c|c|c|}
\hline $\mathrm{N} .^{\circ}$ de silabas & $\bar{x}$ & N. ${ }^{\circ}$ de silabas & $\bar{x}$ \\
\hline 1 & 60,7 & 12 & 5 \\
\hline 4 & 59,5 & 14 & 4 \\
\hline 3 & 51 & 15 & 3,7 \\
\hline 2 y 5 & 47,5 & 16 & 3,6 \\
\hline 7 & 39 & 13 & 2,7 \\
\hline 6 & 38,2 & 18 & 2,3 \\
\hline 8 & 28,5 & 24 & 2 \\
\hline 9 & 20,5 & 17 y 19 & 1,3 \\
\hline 10 & 17,25 & $20,22,23$ & 1 \\
\hline 11 & 11,25 & & \\
\hline
\end{tabular}


El $59,18 \%$ del total de grupos de entonación están formados, y en este orden de mayor a menor, por una, cuatro, tres y dos o cinco silabas: rango paralelo al que obtuvimos en los diálogos dirigidos.

4.2. Las columnas de los cuadros 9 (diálogos dirigidos) y 10 (diálogos espontáneos) representan los siguientes valores:

1. Valor medio del número de sílabas por grupo de entonación.

2. Duración media del grupo de entonación.

3. Duración media de las silabas.

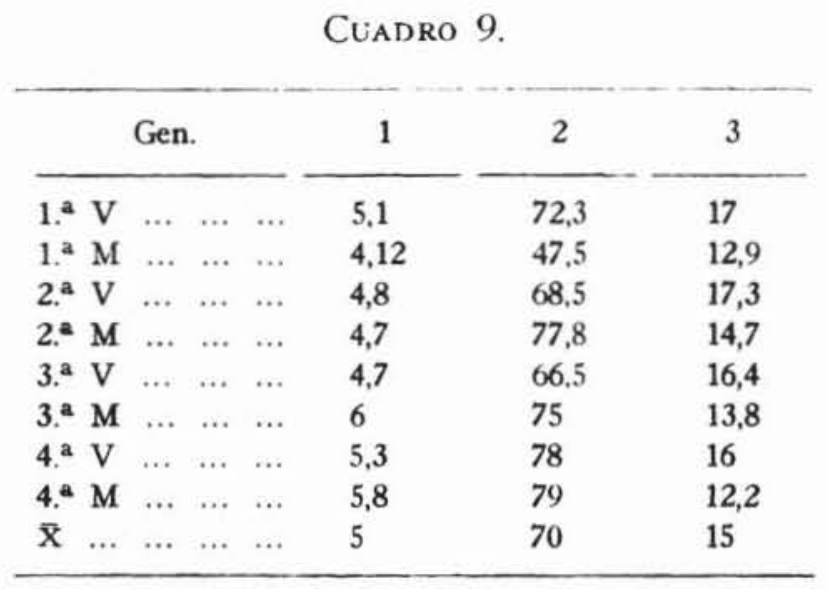

Cuadro 10.

\begin{tabular}{|c|c|c|c|c|}
\hline & Gen. & 1 & 2 & 3 \\
\hline $1 .^{a}$ & $\operatorname{Ve} \ldots \ldots \ldots$ & 5,4 & 76 & 15,8 \\
\hline $1 .^{\mathrm{a}}$ & $\mathrm{Me} \ldots \ldots \ldots$ & 4,9 & 68,1 & 15,7 \\
\hline $4 .^{a}$ & $\mathrm{Vd} \ldots \ldots \ldots$ & 5 & 65,6 & 15,8 \\
\hline 2. ${ }^{a}$ & Md ... ... . & 4.7 & 62,6 & 14,7 \\
\hline$\overline{\mathrm{x}}$ & $\begin{array}{lllll}\ldots & \ldots & \ldots & \ldots\end{array}$ & 5 & 68 & 15,5 \\
\hline
\end{tabular}

Los valores medios son prácticamente iguales en los dos estilos de habla indicados.

La media del número de sílabas en los grupos de entonación es de cinco. La duración media del grupo de entonación se eleva a 69,7 c. s. La duración de las sílabas es de 15,2 c. s.

El valor medio del número de sílabas por grupo de entonación y la duración media de éste, aunque con pocas variaciones, tiene una distribución 
desigual entre los grupos estudiados; por el contrario, la duración media de las silabas es siempre mayor entre los hombres.

\section{Conclusiones.}

En la lengua hablada, los valores correspondientes a los grupos fónicos y a los grupos de entonación son los siguientes:

1. En los grupos fónicos: a) los grupos fónicos más numerosos en los diálogos dirigidos son los de una y cuatro sílabas; en los diálogos espontáneos son los de cinco, seis y cuatro sílabas, en este orden; b) la media del número de sílabas en todos los estilos de habla es de 9,5; los valores medios son mayores en las mujeres que en los hombres; c) la duración media del grupo fónico es de 130,55 c. s. (los valores son ligeramente superiores e inferiores en los diálogos dirigidos y espontáneos, respectivamente): en general, la duración es mayor en las mujeres que en los hombres; d) la duración media de la sílaba es de 15,8 c. s. : este valor es superior en los varones.

2. En los grupos de entonación: a) los grupos de entonación más numerosos están formados por una, cuatro y tres sílabas; b) la media del número de sílabas por grupo de entonación, en todos los estilos de habla es de cinco; c) la duración media del grupo de entonación es de 69,7 c. s.; d) la duración media de las silabas es de 15,2 c. s., valor que coincide, lógicamente, con el análogo de los grupos fónicos. 